days of life it was only 0.9 per cent. (This was one eye only out of 55 observations.)

It would seem therefore that there is some direct connection between the acquirement and use of binocular vision, and the elevation of the eyes as their position of rest.

\title{
REFERENCES
}

1. Johnson, Swan and Weigaud.-In what positions do healthy persons sleep ? Jl. of Amer. Med. Assoc., Vol. XCIV, p. 2058, 1930.

2. Bramwell, E.-The upward movement of the eyes. Brain, Vol. XI, p. 1, 1928.

\section{PIGMENTED DEPOSITS IN THE LENS AND CORNEA OF DOUBTFUL NATURE}

\author{
BY
}

\author{
LT.-Col. R. E. Wright, I.M.S., and DR. K. Koman NaYaR
}

GOVERNMENT OPHTHALMIC HOSPITAL, MADRAS

THE case concerning which this note is a brief record seems sufficiently interesting for publication if only because we have not seen such a condition before and do not know whether it may be regarded as similar in type to the changes met with in chalcosis or pseudo-sclerosis described in the literature. The notes of the case are long and tedious and it is not proposed to deal with them in detail, but rather to start with the description of the condition as it appears now, and then consider points which may be pertinent.

R. T., Hindu, male, aged 17 years, was first seen in 1924 for what seemed to be a mixed condition of trachoma and conjunctivitis eczematosa, and has been under almost continuous observation ever since, with one long interval of over two years1926 to 1928 . At the present time the boy is apparently in the best of health, his eyes are quiet, the conjunctivae smooth, scarring is present but not marked, and the remnants of generalised pannus are easily observed on focal illumination even with the naked eye, but the vessels are pale and bloodless. There is no lacrymation or discharge, but a slight intolerance of glare. The picture, from a water colour painting, gives some idea of the naked eye appearance of the eyes. The corneae present a fairly uniform olivaceous tint, translucent when looked at direct and taking on a more cloudy opalescence as one changes the direction of gaze and views the eye from the side. Looking through the corneae yellowish-brown opacities appear to occupy the pupils. 
On dilatation these are seen to be anterior capsular opacities of rounded shape, some $3 \mathrm{~mm}$. in diameter with chocolate brown, somewhat sinuous borders. The lens as seen outside this border looks practically clear. The condition is almost symmetrical on either side. It is difficult on viewing the eyes in this way to realise that the vision is $6 / 12$ in the right and $6 / 36$ in the leftwith a small correction-for the cataractous appearance is well marked. The pupils are round and active, and their borders in a dull light appear to come to rest just outside the brown rims of the lenticular opacities. In ordinary light the pupils contract to a position overlapping the opacities. With a low power of the corneal microscope and the angle of illumination about 60 degrees, the feature which is most striking, is the shadow of the occluded superficial vessels - the remains of the pannus-thrown on a grey-green opaque looking background apparently coincident with Descemet's membrane. Viewed in such a way the layer of opacification appears uniform and complete throughout the extent of the posterior corneal surface. The cornea in front of this is clear throughout. One gets the impression of a thin, but evenly deposited, paint on the back of a glass. It gives a partial mirror effect, but does not reflect clearly like the fine metallic surface of a modern snow glass. By varying the beam, one is enabled to see the surface of the lens through a slight haze, like viewing a facet through the cloud in an opal. The surface of the lenङ capsule is smooth and the superficial deposit here is of a differen shade, a light brown or a raw sienna colour. This rounded area of opacification is bounded by a relatively wide border of deep chocolate colour. An apparent change of level at this border suggests a slight raising up of the whole opaque area as a shallow plaque. This, however, is not verified by the high power. It is possibly a change in depth, the brown margin being at a slightly deeper level, but more likely to be an optical illusion. The outline of the brown border is undulatory, giving a serpiginous contour to the affected area. Beyond the deep brown edge, which serves to mark off the opacity sharply to the naked eye, a narrow zone of fine haze is seen with the low power and beyond this apparently normal lens. With a change of direction of the beam, it is observed that this central opacity is thin and translucent. The posterior surface of the lens does not show any gross change or posterior sub-capsular opacity. When viewed with lateral illumination, the lens deposit presents a surface even more opaque than the posterior corneal opacification similarly viewed. With the high power, the outline of the corneal endothelium can be made out with a little difficulty under suitable conditions of focusing and illumination, but it is so indistinct as to suggest that the pigment deposit is not in Descemet's membrane but in the endothelium itself, although 


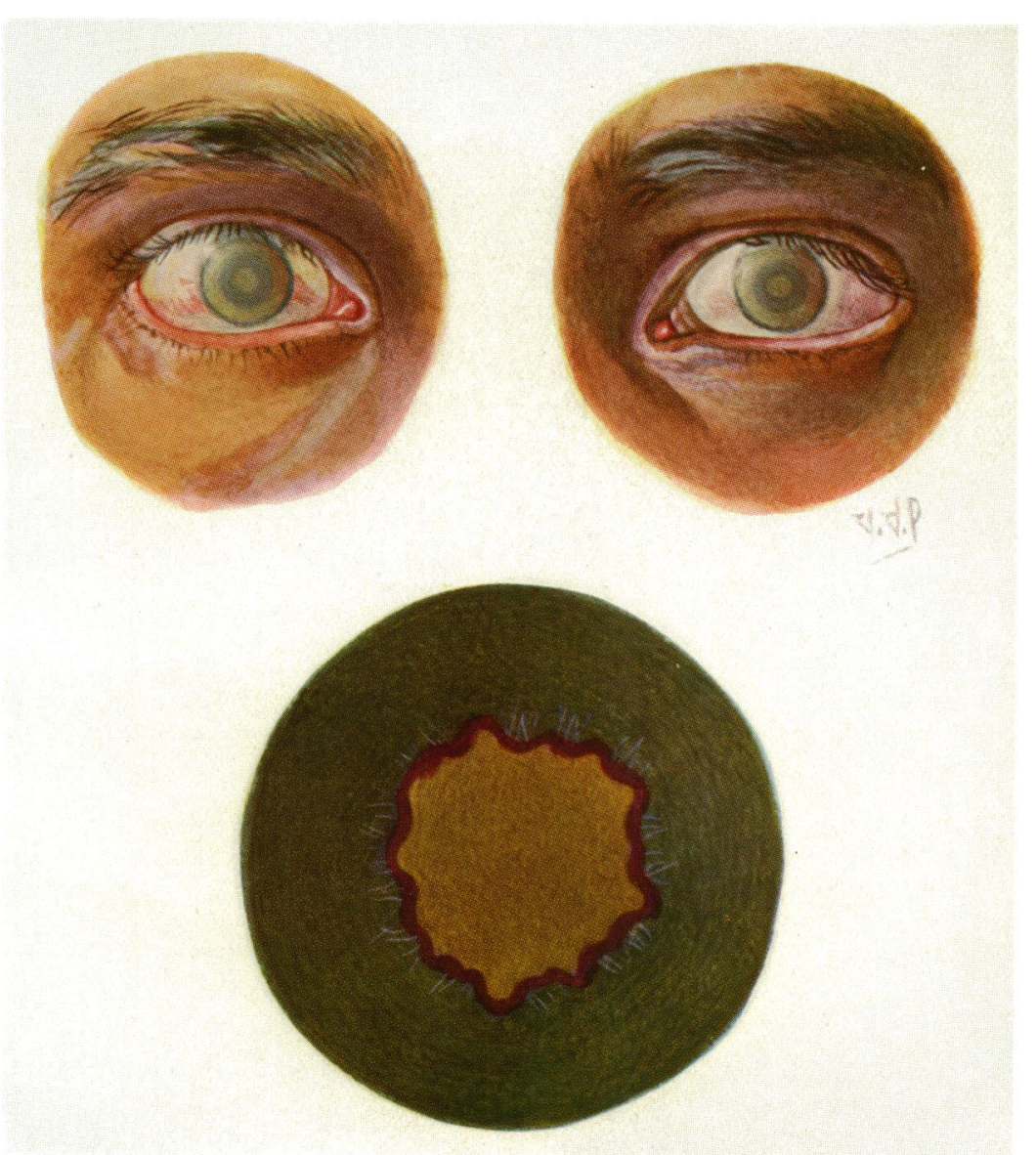

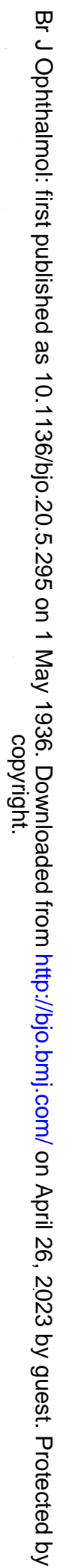


no detail can be resolved to satisfy one that this is so. There are no remains of deep vessels in the cornea. The lenticular opacity presents a few features with the high power not appreciated otherwise. Its texture looks more like a fine metallic deposit of old gold colour. There are fine radiating whitish lines in the superficial layers of the lens surrounding it forming a sort of corona. This is represented in the diagram. In the apparently clear lens beyond-which appears deep amber with a greenish-yellow sheen -it is possible to detect minute yellowish-green points like a fine and very sparse powdering. It is not easy to make out their depth, but some appear to be near the surface and some a short distance beneath the surface.

With the ophthalmoscope, the fundi appear to be within normal limits, although, of course, the view is somewhat muffled. This is sufficient to give a general impression of the condition at present. The patient is the only member of the family affected and the corneal and lenticular appearances have been stationary for the past three years. Before that the lens condition was practically the same, but the corneae were not so much affected.

Some of the main points in the history may now be given, extracted by Dr. N. Pillai, from voluminous notes made in the course of the past 11 years by members of the hospital staff.

"When first seen in December, 1924, at the age of 7 years, the conjunctival condition was very mixed. It gave the impression of being trachomatoid in nature, but there was a decided element of conjunctivitis eczematosa present. There was pannus definitely more marked above. The condition was sufficiently atypical to prevent a positive diagnosis being made. He had been under treatment for trachoma for some time before, which helped greatly to modify whatever condition or conditions were present originally. This mainly consisted in the intermittent application of silver nitrate and copper sulphate with argyrol and zinc drops. Copper treatment apparently had been infrequent and only during the previous year.

After 1924 the history is more complete except for one long period above mentioned. He was at first treated by the usual general and local methods-including mercuric compounds-for conjunctivitis eczematosa, and shortly the lacrymation and photophobia lessened.

In December, 1925, his eyes were much quieter. He had not used copper during this period, but kept up magnesium sulphate washes and mild astringent remedies.

His vision was : right eye, 6/6, with correction ; left eye, 6/6, with correction.

He was advised to continue treatment on these lines. There is no note of the lenticular opacity at this time, although it is 
not at all likely that it could have been missed in the routine examinations with corneal microscope and ophthalmoscope.

He was next seen in March, 1928, when the lenticular opacities were first noted. There was intense lacrymation and photophobia, a trachomatoid inflammatory condition of the palpebral conjunctivae, and generalised pannus more marked in the upper halves of the corneae. The corneae were hazy, oedematous, and thickened. There was a fine deep vascularisation towards the periphery in addition to the pannus, an interstitial keratitis, but no obvious uveitis. As far as could be made out through such corneae, the lenticular opacities looked like "a powdery fluorescent layer just under the capsule." The boy looked decidedly ill. He had enlarged sub-maxillary and sub-mental glands and bleeding gums, a positive tuberculin reaction, but X-ray examination of the chest was negative. The tonsils had been removed some years before. The Wassermann reaction was negative, and there was no evidence of syphilis. He was kept at rest in bed for three weeks and during this period showed a normal temperature chart. His eyes were treated for the most part by lavage (magnesium sulphate), sedative and mydriatic drops. Subsequently, he had light therapy (applied to the skin, not the eyes), cod-liver oil, liver extract, and a course of bacillary emulsion (tuberculin) injections.

During the two years from March, 1928, to March, 1930, hee was frequently an in-patient and various other methods were adopted in addition to the above, including applications of the thermophore to the corneae, small doses of thyroid extract, iodine, irradiated ergosterol internally.

In March, 1929, his general condition was much improved, his mouth was healthy, glandular enlargements had subsided, the weight had increased. The keratitis was much less and lacrymation and photophobia decreased.

Right vision, $6 / 12$; left vision, fingers at three metres.

About this time he developed what appeared to be a malarial attack. It was not a fresh infection. Although the history of previous malarial fever was doubtful, there was every opportunity of his contracting the disease while a child, as he then lived in a heavily infected area. The blood examination was negative, but his symptoms, together with enlargement of the liver and spleen and ascites, led to his being transferred to a general hospital where he had quinine treatment. He rapidly improved and was allowed to go home in October, 1929, to continue eye washes, mydriatics, sub-conjunctival saline injections and dionine. His liver and spleen remained somewhat enlarged and are so at the present time. He was readmitted a month later as the left eye had flared up and the pannus was generalised. From this time the local treatment was changed. He had silver and copper appli- 
cations in early November, 1929, and in the following month radium treatment (about 25 milligramme-hours in all). This was carried out at different sittings by embedding two small curved needles $(0.5 \mathrm{~mm}$. platinum) of 2.5 milligramme each in a paraffin shell, placing this in the eye, closing the lids and leaving it for a variable period up to one hour. A similar method has been used in spring catarrh cases with benefit to the conjunctiva and without apparent harm to cornea or lens.

About January, 1930, the staining of the cornea was first definite. In the right eye-relatively quiet at this time-there was a fringe of superficial vessels extending on to the cornea throughout its periphery for about $4 \mathrm{~mm}$. Between the loops of vessels there was a greenish colouration, and on the deep aspect of the cornea (about the level of Descemet's membrane) a definite olivaceous haze forming a slightly broader belt (say, 5 or $6 \mathrm{~mm}$.). There were no deep vessels seen. The lenticular opacities now showed jagged peripheral radiations in parts, but the outline for the most part was sharp cut and deep brown just as at the present time. The area of the lenticular opacities was practically the same then as now. Up to the time this corneal staining was noted, he had, in all, 33 applications of solid copper sulphate to his conjunctiva whilst under our care.

In February, 1931, right vision, 6/60 ; left vision, 6/24.

During 1931, the eyes were, for the most part, treated by lavage, dionine, atropine or hyoscine, chloretone, and sub-conjunctival saline injections. He had occasional touchings with copper.

In January, 1932, right vision, fingers at 4 metres; left vision, $6 / 24$.

The colouration had now spread throughout the deep surface of the corneae. The note reads : "It is of a pale yellow, absolutely translucent, without any evidence of granularity. The endothelium is not seen, the impression is given that the yellow colour is situated about Descemet's membrane."

From 1932 to 1935 , treatment varied, the changes being rung on lid applications of silver 1 per cent., solid copper sulphate, chaulmoogra oil and lavage. In 1934 the boy had found out for himself what appeared to be best for his eyes and local medication was confined to copper ointments and irrigations.

He has no doubt had a great deal of copper since 1932 in various forms, but the extent of the pigmentation does not seem to have varied much since the observations of early 1932 .

July, 1935 (last note). "The eyes have been quiet now for some months. He has used copper ointment and hypertonic irrigations (magnesium sulphate or sea water) regularly, and is comfortable on this treatment.

Occasionally there is a flare up which is controlled by hot 
compresses, zinc, pantocaine and atropine instillations and further irrigation. He uses goggles, Crookes' B 2. The vision is, as stated above, right eye, $6 / 12$; left eye, $6 / 36 . "$

In seeking a possible explanation of the peculiar colouration and distribution of the pigmentation, it seemed to us that either metallic compounds used in treatment, or broken-down haemoglobin was the most likely source of the pigment which we assume to have been deposited in the corneal endothelium and lens epithelium. There are obvious objections to the former based on records in the literature. Neither silver, copper nor other metallic compounds were used in very unusual quantity before the appearance of the lenticular deposits. That radiant energy (solar, not the radium) may have been a factor is suggested by the distribution, the whole corneal endothelium is affected, but only the lens epithelium unprotected by the iris is involved (at least there has been an apparent protection of the lens under cover of the iris). There has not been any evidence of uveal pigment migration. It is significant, perhaps, that in the breaking up of haemoglobin a variety of coloured products are obtained, the light browns and browns of haemosiderin, the dark browns and bluish-blacks of haematin, the browns of the porphyrins (in the group of hyperporphyrisms there may be photosensitisation), the yellow-brown of haemosiderin, the green of sulphaemoglobinemia, the orange and yellow of bilirubin easily deposited from breaking down red blood corpuscles and its green oxidation product, biliverdin. The light brown and dark brown of the lenticular deposits and the greenish-yellow colouration of the corneal pigmentation in this case is at least suggestive of a common origin from a substance productive of such different pigmentary effects in successive stages of chemical decomposition varying with the site (endothelium or epithelium) and consequently the intensity of radiant energy or chemical action. There certainly was ample opportunity in this case for the local disintegration of haemoglobin.

\section{THE COUNCIL OF BRITISH OPHTHALMOLOGISTS}

\section{Annual Report}

The Council presents its report for the year 1935-36. At its first meeting the following were elected Officers:-President: Sir John Parsons; Vice-Presidents: Mr. P. H. Adams, Mr. E. Brewerton; Hon. Treasurer: Mr. A. H. Levy; Hon. Secrelary: Mr. M. L. Hepburn. 